\title{
Perceptions des frères et des soeurs adultes d'une personne présentant une déficience intellectuelle sur leurs relations mutuelles dans la famille, leurs projets de vie et le soutien souhaité \\ Perceptions of adult siblings of a person with intellectual disability on their mutual relations in the family, their life projects and the expected support
}

\author{
Mariane Villeneuve, Georgette Goupil et Hubert Gascon
}

Volume 23, 2012

URI : https://id.erudit.org/iderudit/1015666ar

DOI : https://doi.org/10.7202/1015666ar

\section{Aller au sommaire du numéro}

Éditeur(s)

Revue francophone de la déficience intellectuelle

ISSN

1929-4603 (numérique)

Découvrir la revue

Citer cet article

Villeneuve, M., Goupil, G. \& Gascon, H. (2012). Perceptions des frères et des soeurs adultes d'une personne présentant une déficience intellectuelle sur leurs relations mutuelles dans la famille, leurs projets de vie et le soutien souhaité. Revue francophone de la déficience intellectuelle, 23, 104-120. https://doi.org/10.7202/1015666ar

\section{Résumé de l'article}

Peu d'études examinent les relations dans la fratrie adulte lorsqu'un membre de la famille présente une déficience intellectuelle (DI). Cette étude a pour objectif d'explorer les perceptions des frères et des soeurs adultes d'une personne présentant une DI sur l'influence de cette personne à l'égard de leurs projets de vie. Elle vise aussi à décrire les perceptions des fratries sur les comportements et les habiletés de la personne présentant une DI, leurs perceptions des relations fraternelles dans la famille et le soutien désiré dans ce contexte. Treize frères et soeurs adultes d'une personne ayant une DI ont répondu, en entrevue, à un questionnaire validé par des experts. L’analyse révèle que les participants s'impliquent dans la vie familiale, en regard de la déficience, en soutenant leurs parents et la personne présentant une DI. Enfin, plusieurs mentionnent que la déficience de leur frère ou de leur soeur influence leurs projets de vie, surtout aux plans de l'orientation professionnelle, du lieu de résidence futur et de l'éducation de leurs enfants. La conclusion présente des recommandations cliniques quant aux mesures de soutien destinées aux familles.
Tous droits réservés @ Revue francophone de la déficience intellectuelle, 2012
Ce document est protégé par la loi sur le droit d'auteur. L’utilisation des services d'Érudit (y compris la reproduction) est assujettie à sa politique d'utilisation que vous pouvez consulter en ligne. 
REVUE FRANCOPHONE DE LA

DÉFICIENCE INTELLECTUELLE

VOLUME 23, 104-120

\title{
PERCEPTIONS DES FRÈRES ET DES SEURS ADULTES D'UNE PERSONNE PRÉSENTANT UNE DÉFICIENCE INTELLECTUELLE SUR LEURS RELATIONS MUTUELLES DANS LA FAMILLE, LEURS PROJETS DE VIE ET LE SOUTIEN SOUHAITÉ
}

\author{
Mariane Villeneuve, Georgette Goupil et Hubert Gascon
}

\begin{abstract}
Peu d'études examinent les relations dans la fratrie adulte lorsqu'un membre de la famille présente une déficience intellectuelle (DI). Cette étude a pour objectif d'explorer les perceptions des frères et des sœurs adultes d'une personne présentant une DI sur l'influence de cette personne à l'égard de leurs projets de vie. Elle vise aussi à décrire les perceptions des fratries sur les comportements et les habiletés de la personne présentant une DI, leurs perceptions des relations fraternelles dans la famille et le soutien désiré dans ce contexte. Treize frères et sœurs adultes d'une personne ayant une DI ont répondu, en entrevue, à un questionnaire validé par des experts. L'analyse révèle que les participants s'impliquent dans la vie familiale, en regard de la déficience, en soutenant leurs parents et la personne présentant une DI. Enfin, plusieurs mentionnent que la déficience de leur frère ou de leur sœur influence leurs projets de vie, surtout aux plans de l'orientation professionnelle, du lieu de résidence futur et de l'éducation de leurs enfants. La conclusion présente des recommandations cliniques quant aux mesures de soutien destinées aux familles.
\end{abstract}

\section{INTRODUCTION}

Les membres de la famille offrent différents types de soutien, tout au long de la vie, aux personnes la composant. La famille est un système qui évolue avec le temps: formation du couple, naissance, enfance puis adolescence, départ des enfants de la maison familiale, vieillissement et décès des parents. Ces étapes ne suivent pas forcément cet ordre et des évènements imprévus viennent souvent les bouleverser. Pour une famille dont un enfant présente une DI, le vieillissement des parents est une source d'inquiétudes, dans la mesure où ce sont généralement eux qui prennent soin de la personne ayant des besoins spéciaux (Holmes et Carr, 1991).

Mariane Villeneuve, étudiante au doctorat en psychologie, Université du Québec à Montréal ; Georgette Goupil, professeure au département de psychologie, Université du Québec à Montréal ; Hubert Gascon, professeur au département des sciences de l'éducation, Université du Québec à Rimouski.
Cet article présente d'abord l'influence du vieillissement des membres des familles où une personne présente une DI et certains constats, issus d'écrits scientifiques, au sujet des fratries. Suite aux précisions concernant la méthode employée, suivent les résultats de l'étude. En conclusion, les apports et les limites de l'étude sont soulignés.

\section{$\underline{\text { Famille, fratrie et déficience intellectuelle }}$}

Rizzolo, Hemp, Braddock et Pomeranz-Essley (2004) indiquent qu'au cours des dernières années, le gouvernement américain a augmenté considérablement les subventions, dont une partie va à des mesures de soutien aux familles, afin de favoriser les choix individuels des personnes ayant un retard de développement (incluant la DI). Selon des données américaines, 73,6 \% des adultes ayant un retard de développement vivent avec un membre leur famille (Fujiura, 2010). Cela est en lien avec les politiques en faveur du maintien des personnes présentant une DI dans leur milieu naturel. En effet, alors que ces

Volume 23, 2012 
personnes étaient autrefois institutionnalisées, les politiques à ce sujet favorisent maintenant leur maintien dans leurs familles. Avec l'accroissement de leur espérance de vie, les personnes ayant une DI demandent des services sur une plus longue période de temps. Le vieillissement et ses conséquences sur l'autonomie exigent que l'on développe d'autres types de services, plus adaptés à cette nouvelle réalité (Seltzer, Greenberg, Krauss, Gordon et Judge, 1997). Dans ce contexte, la fratrie est souvent considérée par les mères comme la ressource qui prendra, en partie du moins, la relève des parents (Bernard, 2011).

La fratrie est constituée des frères et sœurs d'une même famille. Rapportant les travaux de Cicirelli, Ansen-Zeder (2010) souligne la variété des situations associées à la définition de ce terme. Ainsi, les frères ou les sœurs peuvent être les enfants des mêmes parents biologiques ou être des enfants adoptés par un couple de parents. Avec les divorces et la reconstitution des familles, il peut aussi s'agir de demi-frères ou demi-sœurs n'ayant en commun qu'un seul parent biologique. La fratrie peut aussi être formée de beaux-frères et de belles-sœurs, fils ou filles d'un partenaire conjugal, mais qui n'ont aucun lien de sang entre eux. Enfin, il peut s'agir d'une personne qui est considérée, à cause de sa proximité affective avec la famille, comme un frère ou une soeur (Ansen-Zeder, 2010). Les parents ne sont donc pas nécessairement les parents biologiques et ces frères et ces sœurs ne sont pas toujours unis par les liens du sang (Oris, Brunet, Widmer et Bideau, 2007). Les relations fraternelles sont, selon Cicirelli (1995), les plus durables qu'un individu développe au cours de sa vie. La fratrie passe par certaines étapes et remplit certaines fonctions, selon l'âge de ses membres (Turnbull, Turnbull, Erwin et Soodak, 2006).

Les études portant sur les frères et les sœurs d'une personne présentant une DI ont d'abord porté sur l'enfance et les conséquences négatives engendrées par la DI (Hodapp, Glidden et Kaiser, 2005). Il semble maintenant que les études sur le sujet présentent aussi les conséquences positives de cette situation (Doody, Hastings, O'Neil et Grey, 2010; Kao, Romero-Bosch, Plante et Lobato, 2011). Plusieurs publications concernent, pour les périodes de l'enfance et de l'adolescence, les fratries des personnes qui ont une déficience intellectuelle. Ces publications présentent, entre autres, comment utiliser des groupes de soutien pour aider ces fratries (Scelles, 2007).

Les études menées auprès de cette population adulte permettent de faire certains constats. Selon Seltzer, Greenberg, Orsmond et Lounds (2005), la recherche indique que les fratries auront un niveau d'implication élevé auprès de la personne tout au long de leur vie. Cette implication peut aller jusqu'à la cohabitation avec la personne ayant une DI. Les fratries évaluent leur implication de façon plutôt positive, comparativement aux frères et aux sœurs de personnes ayant un problème de santé mentale, qui évaluent leur implication de façon plutôt négative. Les frères et les sœurs perçoivent une grande influence de la présence d'une personne présentant une DI et vivent avec la connaissance de la déficience dès l'enfance (Seltzer, Begun, Seltzer et Krauss, 1991). Ainsi, les frères et les sœurs sont exposés tôt aux demandes engendrées par la déficience. Cependant, les parents sont parfois réticents à demander un grand soutien aux autres enfants de la famille. Ainsi, les mères vieillissantes abordent peu le sujet de leur relève auprès de la personne ayant une DI avec les fratries qui doivent, dans plusieurs cas, prendre l'initiative de cette discussion (Bernard et Goupil, 2012).

Selon Orsmond et Seltzer (2000), les relations fraternelles se caractérisent par des échanges fréquents et des relations rapprochées. Toutefois, Taylor, Greenberg, Seltzer et Floyd (2008) soulignent que ces relations s'établissent surtout lorsque la fratrie apporte une aide fonctionnelle (exemples : soutien financier, préparation des repas, transport) à la personne ayant une DI. Plusieurs auteurs notent que les sœurs s'impliquent généralement plus que les frères (Burke, Taylor, Urbano et Hodapp, 2012; Coniglio et Meldrum, 2011; Krauss, Seltzer, Gordon et Friedman, 1996; Orsmond et Seltzer, 2000). À partir d'une étude par entrevue auprès de 12 sœurs de femmes ayant une déficience intellectuelle, Wilson (2011) note que les projets de vie de ces sœurs sont influencés par la perspective des responsabilités futures.

Bien que le domaine suscite de plus en plus d'intérêt avec le vieillissement de la population, les fratries adultes dans lesquelles une personne présente une DI ont fait l'objet de moins d'études que celles menées 
auprès des enfants (Orsmond et Seltzer, 2007; Villeneuve, Goupil et Gascon, 2011). Actuellement, les frères et les sœurs deviennent des ressources importantes pour les familles surtout lorsque les parents ne peuvent plus assumer les soins de la personne présentant une DI (Davys, Mitchell et Haigh, 2011). Il appert donc qu'une meilleure connaissance des perceptions des fratries dans lesquelles une personne présente une DI permettrait de mieux cerner les besoins et d'aider les familles.

\section{Objectifs de l'étude}

Cette étude exploratoire a pour objectif de décrire les perceptions des frères et des sœurs adultes d'une personne présentant une DI et l'influence de cette personne sur les projets de vie de la fratrie. Cette étude vise aussi à décrire les perceptions des fratries sur les comportements et des habiletés de la personne présentant une DI, les relations fraternelles dans la famille et, le cas échéant, le soutien désiré dans ce contexte.

\section{MÉTHODE}

\section{Participants et procédure de recrutement}

Les participants recrutés devaient respecter certains critères : avoir un frère ou une sœur qui présente une DI, non associée à un trouble envahissant du développement, et qui habite toujours la maison familiale ; être âgés entre 20 et 35 ans ; avoir un écart d'âge d'un maximum de 8 ans avec la personne présentant une DI ; et ne plus habiter la maison familiale depuis au moins un an. Les participants ont été recrutés par le biais d'annonces publicitaires décrivant le projet de recherche et placés dans des associations en déficience intellectuelle.

\section{Le frère ou la sœur d'une personne présentant une DI}

Sept frères et six sœurs d'une personne présentant une DI collaborent à cette étude. Ces frères et ces sœurs ont les mêmes parents naturels que les personnes ayant une déficience intellectuelle. Âgés de 20,18 à 34,44 ans $(M=26,34, E ́ T=3,70)$, ils ont un écart d'un maximum de 7 ans avec la personne présentant une DI. Ils proviennent de différentes régions du Québec: Capitale-Nationale $(n=2)$,
Lanaudière $(n=1)$, Laurentides $(n=1)$, Montérégie $(n=2)$ et Montréal $(n=7)$. Un seul participant a des enfants. Le Tableau 1 présente les caractéristiques des participants.

\section{La personne présentant une DI}

Les personnes présentant une DI sont âgées entre 16,35 ans et 32,94 ans $(M=24,09, E ́ T=5,39)$. Pour s'assurer du diagnostic de DI, étant donné l'impossibilité d'avoir accès aux dossiers, les personnes doivent respecter certains critères. D'abord, 10 des 13 participants ont un frère ou une sœur qui reçoit ou a reçu des services d'un centre de réadaptation en déficience intellectuelle et troubles envahissants du développement (CRDITED). Étant donné que l'accès à ces services repose sur les critères de la DI, tels que définis par l'American Association on Intelllectual and Developmental Disabilities (AAIDD) (Luckasson et al., 2002/2003), il a été déduit que ces personnes présentent une DI. Ensuite, les trois autres participants ont un frère ou une sœur présentant un syndrome particulier entraînant une DI, confirmé par les parents (syndrome de Down, agénésie du corps calleux et sclérose tubéreuse). En ce qui concerne le niveau de sévérité de la DI, 5 participants ne le connaissent pas. Les autres présentent, selon les participants, une DI légère $(n=2)$, moyenne $(n=5)$ ou grave $(n=1)$. Au primaire, leur scolarisation a eu lieu en classe ordinaire $(n=7)$ ou en classe spéciale $(n=5)$. Au secondaire, la scolarisation a eu lieu en classe spéciale pour tous ceux qui fréquentent ou ont fréquenté l'école $(n=12)$. Une personne n'a pas été scolarisée, tant au primaire qu'au secondaire. Sur les 26 parents biologiques, 23 sont d'origine caucasienne et trois d'origine arabe. Quatorze sont toujours en couple et 12 sont séparés.

\section{Instruments}

Une fiche signalétique, consignant des données concernant le participant, sa fratrie et ses parents, et un protocole d'entrevue, élaborés spécifiquement pour cette étude, servent à recueillir les données. Le protocole d'entrevue, construit en fonction de huit thèmes de recherche, comprend 30 questions et 10 sous-questions. Il explore les perceptions des participants relativement (a) à leur frère ou leur sœur présentant une DI, (b) aux relations dans la fratrie, 
(c) à leur implication dans leur famille, (d) à l'acceptation de leurs parents à l'égard du diagnostic, (e) à l'influence de leur situation sur certains de leurs choix de vie, (f) aux évènements marquants à l'intérieur de la fratrie, (g) à leurs propres besoins de soutien et (h) à leurs suggestions pour les membres des familles et pour les services. Sept experts, dans le domaine de la recherche qualitative, de la famille ou de la DI, valident le contenu de ces deux instruments et ils transmettent leurs commentaires grâce à une grille d'évaluation. Après les modifications, les instruments sont préexpérimentés auprès de trois sœurs de personnes présentant une DI, avant leur utilisation auprès des participants.

\section{$\underline{\text { Tableau } 1}$}

Caractéristiques démographiques des participants $(N=13)$

\begin{tabular}{|c|c|}
\hline Caractéristiques & $n$ \\
\hline \multicolumn{2}{|l|}{ Position dans la fratrie } \\
\hline Plus âgé que la personne présentant une DI & 10 \\
\hline Plus jeune que la personne présentant une DI & 3 \\
\hline \multicolumn{2}{|l|}{ Taille de la fratrie } \\
\hline Deux membres & 6 \\
\hline Trois membres & 2 \\
\hline Quatre membres & 5 \\
\hline \multicolumn{2}{|l|}{ État civil } \\
\hline Conjoint de fait & 4 \\
\hline Célibataire & 7 \\
\hline Marié & 2 \\
\hline \multicolumn{2}{|l|}{ Revenu } \\
\hline Moins de 9999 \$/ année & 2 \\
\hline Entre 10000 et 29000 \$/année & 3 \\
\hline Entre 30000 et 49000 \$/année & 7 \\
\hline Entre 50000 et 69000 \$/année & 0 \\
\hline Plus de 70000 \$/année & 1 \\
\hline \multicolumn{2}{|l|}{ Niveau de scolarité (complété ou en cours) } \\
\hline Secondaire & 2 \\
\hline Collégial & 2 \\
\hline Universitaire & 9 \\
\hline \multicolumn{2}{|l|}{ Distance des lieux de résidence des fratries } \\
\hline Moins de 15 km & 6 \\
\hline De 15 à 50 km & 2 \\
\hline De 50 à $100 \mathrm{~km}$ & 3 \\
\hline Plus de $100 \mathrm{~km}$ & 2 \\
\hline
\end{tabular}

\section{Procédure d'entrevue}

Les entrevues se déroulent, selon le choix du participant, à son domicile ou dans un local de l'Université du Québec à Montréal (UQAM). L'expérimentatrice procède à l'entrevue, après l'explication et la signature d'un formulaire de consentement approuvé par un comité éthique de
l'UQAM. Des bandes audio permettent l'enregistrement des entrevues afin d'en faire la retranscription.

\section{Mode d'analyse}

Les données obtenues grâce aux questions fermées de la fiche signalétique permettent une analyse descriptive de type quantitatif : fréquence, moyenne et pourcentage des différentes catégories de 
réponses. Les réponses aux questions de l'entrevue, quant à elles, sont analysées selon la méthode qualitative de Miles et Huberman (2003). Une grille d'analyse élaborée en fonction des catégories choisies, d'après la documentation sur le sujet et après une lecture attentive des comptes-rendus textuels des entrevues, permet une codification des catégories communes abordées par les participants. Ce codage, effectué indépendamment par l'expérimentatrice et par une assistante de recherche, atteint un niveau d'accord inter juge de valeur kappa de Cohen entre 0,80 et $1,00(M=0,95, E ́ T=0,06)$. La section Résultats présente les données en fonction des huit thèmes de recherche ${ }^{1}$.

\section{RÉSULTATS}

\section{Les personnes présentant une DI}

En première partie d'entrevue, le participant répond à des questions relativement à ses perceptions de l'autonomie et des comportements de la personne présentant une DI. Sur le plan de l'autonomie, 6 participants décrivent leur frère ou leur sœur comme assez autonome, 3 expliquent qu'il ou elle est un peu autonome et 3 autres indiquent peu autonome. Un seul participant perçoit la personne présentant une DI comme très autonome.

Selon 9 participants, leur frère ou leur sœur présente des difficultés au plan du comportement. De ceux-ci, 3 mentionnent des problèmes avec une consommation excessive de nourriture et 2 , des problèmes en lien avec des routines rigides. Deux participants précisent que la personne présentant une DI adopte, à l'occasion, des comportements violents : "Il est violent des fois, soit envers les meubles ou envers les personnes ». Finalement, 3 remarquent des difficultés au plan des relations interpersonnelles et 3 autres indiquent que leur frère ou leur sœur vit ses émotions de manière extrême. Un participant explique: "C'est plus dans sa gestion de ses émotions. Donc, à un moment, il est surexcité et tout va être gros, il va parler fort et il va débarrasser [déplacer] de l'air. En même temps, il est beaucoup

${ }^{1}$ Le masculin est employé tout au long du texte dans le but de conserver la confidentialité des participants et de leur famille. comédien. C'est-à-dire, quand il ne se sent pas bien, il va être vraiment écrasé ».

\section{Les relations dans la fratrie}

La deuxième partie de l'entrevue explore les contacts dans la fratrie et les relations fraternelles. La fréquence des contacts dans la fratrie satisfait les 13 participants. De ceux-ci, 10 sont en contact, en personne, avec la personne présentant une DI d'une à plusieurs fois par semaine. Un participant explique: «On a une certaine solidité dans la famille et j'ai besoin de voir mes frères et sœurs. Donc, c'est sûr que j'ai besoin de le voir lui autant que les autres [frères] dans le fond ». Ces contacts fraternels se produisent surtout dans le cadre de visites, de soupers et de fêtes de famille $(n=9)$. Sept participants insistent sur l'amour fraternel qui sous-tend le désir de rencontrer la personne présentant une DI. Deux autres évoquent l'importance qu'a eue l'éducation reçue de leurs parents pour le maintien de bonnes relations fraternelles: "Je pense que c'est beaucoup l'éducation de la part des parents. Le fait aussi qu'il habite chez mes parents, ça doit sûrement aider, mais ça revient surtout à l'éducation». Quatre participants, en particulier ceux habitant loin de leurs parents, mentionnent que, lors des visites, les contacts peuvent durer plusieurs jours. Les 2 participants demeurant à plus de $100 \mathrm{~km}$ de leur fratrie visitent la famille moins souvent, soit de deux à six fois par an.

La fréquence des contacts téléphoniques ou par Internet varie d'une fratrie à l'autre : plusieurs fois par semaine $(n=2)$, une fois par semaine $(n=3)$, une ou deux fois par mois $(n=2)$, une fois par mois $(n=1)$, à tous les deux ou trois mois $(n=1)$, deux ou trois fois par année $(n=1)$ ou jamais $(n=3)$. Tous les participants ayant des contacts de cette nature se disent satisfaits de leur fréquence. Les participants ayant répondu ne jamais avoir de contact par téléphone ou par Internet expliquent leur réponse par les habiletés limitées de communication de la personne présentant une DI.

Une question de l'entrevue porte sur les types de relations partagées dans la fratrie. Le Tableau 2 présente les cinq choix de réponses proposés ainsi que la fréquence des réponses des participants. Plusieurs participants $(n=7)$ expriment toutefois 
leurs difficultés à ne faire qu'un seul choix, ils indiquent donc deux choix de réponse.

Il est intéressant de noter qu'aucun participant n'associe ses relations à indifférence ou ressentiment. Tel qu'abordé précédemment, 4 perçoivent, chez la personne présentant une DI, des problèmes au plan comportemental ou affectif (violence et émotions extrêmes). Trois de ces 4 participants identifient, au moins en partie, leurs relations au type responsabilité, présence en temps de crise et moins d'implication psychologique, soit le type de relations le moins positif identifié par les participants.

\section{$\underline{\text { Tableau } 2}$}

Relations partagées dans la fratrie, selon les participants $(N=13)$

\begin{tabular}{lc}
\hline Types de relations & $n$ \\
\hline Type 1 : attachement profond, confidences, empathie, compréhension mutuelle & 1 \\
Type 2 : amitié, soutien, implication & 4 \\
Type 1 et type 2 & 4 \\
Type 3 : responsabilité, présence lors de crise, moins d'implication psychologique & 1 \\
Type 2 et type 3 & 3 \\
Type 4 : indifférence, pas de soutien & 0 \\
Type 5 : ressentiment, aucun contact & 0 \\
\hline
\end{tabular}

Six participants ont un rôle formellement défini auprès de leur frère ou de leur sœur présentant une DI, soit celui de tuteur ou de fiduciaire, et ils s'en disent satisfaits. Un participant explique : «C'est moi qui le prendrais en charge si mes parents venaient à décéder. Pas pour en prendre soin nécessairement, pas pour l'avoir avec moi, mais pour gérer ses responsabilités». En moyenne, les participants ayant un rôle formel ont 28,14 ans et ceux n'ayant pas de rôle formel ont 29,06 ans. Les personnes présentant une DI dont la fratrie occupe un rôle formel ont en moyenne 26,74 ans alors que ceux pour qui aucun rôle formel n'est attribué à la fratrie ont en moyenne 21,83 ans. Le Tableau 3 présente le nombre de participants, selon leur sexe, ayant ou non un rôle formel auprès de la personne présentant une DI.

\section{Tableau 3}

Nombre de participants ayant ou non un rôle formel auprès de la personne présentant une DI $(N=13)$

\begin{tabular}{cccc}
\hline Sexe du participant & Ayant un rôle formel & N'ayant pas de rôle formel & Total \\
\hline Femme & 3 & 3 & 6 \\
Homme & 3 & 4 & 7 \\
\hline Total & 6 & 7 & 13 \\
\hline
\end{tabular}

\section{$\underline{\text { L'implication dans la famille }}$}

Étant donné l'influence des parents dans les relations fraternelles (Griffiths et Unger, 1994; Zetlin, 1986), la troisième partie de l'entrevue explore l'implication du participant par rapport à sa fratrie et ses parents, la perception des attentes de ses parents ainsi que l'influence positive ou négative d'être le frère ou la sœur d'une personne présentant une DI.
Tout d'abord, 9 des 13 participants mentionnent aider leur frère ou leur sœur dans ses activités.

L'aide prend la forme de gardiennage $(n=4)$, de soins généraux tels que faire à manger, donner un bain ou faire le ménage $(n=4)$, de transport $(n=2)$, d'accompagnement dans les loisirs $(n=4)$ ou d'aide émotionnelle $(n=1)$. Pour 8 des 9 participants, cette aide les satisfait. Un de ceux-ci explique : «Je pense que c'est bien que j'ai une implication, mais trop... 
tu sais, ce n'est pas mon enfant non plus. C'est plus le travail de mes parents, mais quand ça doit arriver, ça arrive puis il n'y a pas de problème, ce n'est pas une corvée ».

Quatre participants disent ne rien faire de particulier pour aider leur frère ou leur sœur ayant une DI. Un explique que la personne ayant une DI n'a pas besoin d'aide, deux précisent que les parents s'en chargent et un dernier mentionne que l'éloignement géographique l'empêche d'aider. Leur implication les satisfait tout de même, soit parce qu'ils ne peuvent pas faire plus pour le moment, soit parce que les parents répondent déjà aux besoins de la personne présentant une DI.

Les participants s'impliquent également auprès de leurs parents. Ils leur offrent une aide pour les soins à la personne présentant une DI $(n=10)$, de l'écoute et des discussions $(n=2)$ ou du transport $(n=3)$. Deux sollicitent le moins possible leurs parents pour leur laisser plus de temps pour s'occuper de la personne présentant une DI. Deux autres ne font rien de particulier pour le moment, mais se disent prêts à aider le moment venu. Leur implication actuelle auprès de leurs parents satisfait l'ensemble des participants. À ce sujet, ils indiquent qu'ils aiment aider et prendre soin des autres $(n=6)$, que cela leur permet de garder contact avec les membres de leur famille $(n=3)$ ou que cela leur a permis d'être autonomes $(n=3)$.

Plusieurs participants croient que leur implication auprès de leurs parents est différente des personnes vivant dans une famille typique $(n=9)$. De ceux-ci, 8 soulignent qu'ils n'auraient pas à fournir d'aide si leur frère ou leur sœur n'avait pas de DI : «Si mon frère n'avait pas de déficience, mes parents pourraient partir quand ils voudraient, ils auraient plus de liberté et moi je n'aurais pas besoin de garder ». Deux autres indiquent qu'ils ne seraient pas aussi proches de leurs parents : «Je pense que ça nous permet de communiquer un peu. Je pense que le moment de la semaine où je parle le plus à ma mère, c'est quand on s'occupe de mon frère ».

Six participants croient que leurs parents s'attendent à recevoir de l'aide d'eux : «Mes parents sont très conscients que je les aide comme je peux puisque je fais des efforts pour les aider. Ils me le demandent quand ils ont besoin, mais quand j'ai autre chose de prévu, je ne vais pas me faire blâmer parce que je peux pas garder ». Les autres mentionnent que leurs parents s'attendent à une présence $(n=3)$ ou à ce qu'ils prennent leur relève en temps opportun $(n=3)$. Deux indiquent toutefois que les attentes de leurs parents leur sont inconnues. Pour la majorité des participants $(n=10)$, les attentes de leurs parents correspondent à leurs désirs.

Huit participants soulignent vivre des désagréments en lien avec leur rôle de frère ou de sœur d'une personne présentant une DI. Certains regrettent de ne pas vivre de relations fraternelles normales $(n=4)$ : "C'est certain que d'avoir un frère, comme appui, un petit frère ou un grand frère, c'est toujours pratique, dans la vie de tous les jours [...] si j'ai besoin d'aide je ne peux pas faire appel à lui». Deux soulignent qu'il est difficile d'être étiqueté comme «le frère de la personne présentant une DI » et 2 autres indiquent que cela restreint les activités familiales et rend certaines situations complexes.

Douze participants mentionnent que d'être le frère ou la sœur d'une personne présentant une DI leur apporte quelque chose de positif. Ils évoquent les joies de la vie quotidienne $(n=3)$, avoir rencontré des gens grâce à la personne présentant une DI $(n=2)$, mais surtout une plus grande ouverture d'esprit et une croissance personnelle $(n=11)$. Un d'entre eux explique, au sujet des joies de la vie quotidienne : "Il me fait réaliser souvent des petites joies de la vie, des petites choses simples. Lui, il est heureux tout le temps. S'il a de la bouffe, du monde autour et des activités, il n'a pas de casse-tête, mon frère. Donc, ça me fait prendre le temps, des fois, de me rappeler de relaxer, juste d'être heureux ».

\section{L'implication et l'acceptation des parents relativement à la DI}

Tous expliquent que leurs parents acceptent maintenant bien la DI. Huit répondent également que l'acceptation de leurs parents par rapport à la DI les influence. En général, cela les incite à voir la DI de la même façon que leurs parents : "Tu sais, il n'y a jamais eu de problème avec ça. On l'accepte. Mes parents l'acceptent donc, oui, je l'accepte aussi. [...] Si mes parents l'avaient mal acceptée, peut-être que je l'aurais mal acceptée ». 
Deux participants ont travaillé dans des associations œuvrant dans le domaine de la DI et 4 autres y ont fait du bénévolat. C'est donc dire que 6 d'entre eux ont déjà collaboré, de près ou de loin, à une association. Le Tableau 4 présente le nombre de participants et de leurs parents dans le mouvement associatif.

\section{$\underline{\text { Tableau } 4}$}

\section{Nombre de parents et de participants impliqués dans le mouvement associatif}

\begin{tabular}{cccc}
\hline $\begin{array}{c}\text { Implication dans le mouvement } \\
\text { associatif }\end{array}$ & Parents impliqués & Parents non impliqués & Total \\
\hline Participant impliqué & 4 & 2 & 6 \\
Participant non impliqué & 4 & 3 & 7 \\
\hline Total & 8 & 5 & 13 \\
\hline
\end{tabular}

Des 8 participants dont les parents ont été ou sont impliqués, 4 croient que cela les influence. De ces 4 personnes, 3 contribuent à des associations et la quatrième explique qu'elle aimerait bien s'y engager dans le futur. Un participant impliqué précise : «En voyant mes parents qui participent à des activités et qui rencontrent des gens qui vivent la même chose qu'eux, j'ai eu envie de le vivre aussi [...] voir s'ils vivaient la même chose que moi et voir si on était pareil ».

$\underline{\text { L'influence sur leurs choix de vie : carrière, lieu }}$ de résidence et enfants

Cinq participants sur treize occupent un emploi ou étudient dans un domaine en lien avec la relation d'aide ou l'enseignement. Lorsqu'on leur demande si avoir un frère ou une sœur ayant une DI influence leur choix de vie professionnelle, 6 répondent oui, 4 répondent non et 3 répondent à la fois oui et non. De ceux répondant par l'affirmative ou à la fois oui et non $(n=9), 4$ désirent travailler dans un domaine qui permet d'aider ou de défendre des personnes et 3 expriment que les apprentissages en lien avec la personne présentant une DI orientent leur choix : "Tu sais, si je n'avais pas un frère handicapé, je pense qu'on ne les voit pas souvent nécessairement [...], donc, peut-être que je ne serais jamais allé làdedans si je n'avais pas connu ». Deux participants ont désiré travailler en relation d'aide, mais ont changé d'avis par la suite.

$\mathrm{Au}$ sujet de leur lieu de résidence, 4 participants mentionnent que la personne présentant une DI influence l'emplacement actuel de leur domicile. Ils choisissent d'habiter près pour aider leur famille et la personne présentant une DI. Trois autres expliquent que la proximité géographique n'est pas nécessaire ou désirée pour l'instant. Deux choisissent d'habiter une région pour les études et 3 n'expliquent pas leurs raisons. Toutefois, lorsqu'on leur demande si la DI de leur frère ou de leur sœur pourrait avoir une influence sur leurs futurs déplacements, tels un déménagement ou des périodes de vacances, dix participants répondent par l'affirmative: "C'est sûr que j'essaierai tout le temps d'habiter dans le coin. Puis même si ça influence mes objectifs de vie, je veux [habiter près]. ».

Lorsqu'on aborde le désir d'avoir des enfants, 2 perçoivent une influence négative de la présence de la personne présentant une DI : «Je veux des enfants ça c'est sûr, mais des enfants qui ont une déficience, je ne sais pas encore si je les garde. L'avortement, ça me donnerait l'impression de dire à mon frère qu'il n'aurait pas le droit de vivre». Onze participants croient que d'avoir été en contact avec une personne présentant une DI modifiera leur manière d'éduquer leurs enfants. Ils expriment surtout le désir que leurs enfants aient l'esprit ouvert à la différence $(n=8)$. Le Tableau 5 présente des extraits de verbatim à cet effet.

Le Tableau 6 présente, pour chacun des participants, quels projets de vie ont été influencés par la présence d'un frère ou d'une sœur ayant une déficience intellectuelle. 


\section{Les évènements marquants dans la fratrie}

La sixième partie d'entrevue est centrée sur les principaux évènements de vie significatifs dans les relations fraternelles. Ces évènements peuvent être de nature positive ou négative. Onze participants indiquent à la fois des évènements positifs et négatifs et 2 n'indiquent que des évènements positifs. Pour 5 participants, les évènements négatifs sont en lien avec la santé de la personne présentant une DI : «Il a déjà passé 4 ou 5 jours à l'hôpital aux soins intensifs. Probablement que j'ai trouvé ça plus difficile que lui parce que lui, il n'avait pas conscience de ce qui se passait ». Quatre indiquent des évènements en lien avec la vie scolaire, soit des difficultés d'intégration scolaire et de l'intimidation par les pairs : «Un souvenir qui reste, c'est quand j'ai su que mon frère s'était fait ridiculiser à l'école. Il avait été humilié devant plein de monde. Donc ça, ça a eu un impact, je pense que j'ai développé de la colère ». Trois mentionnent des difficultés au plan social. Par exemple, 1 exprime la difficulté de vivre avec le regard des autres lors de sorties familiales. Finalement, 2 autres expliquent qu'il est pénible pour les membres de la famille de remarquer la différence de la personne présentant une DI : «Je me suis rendu compte à quel point il avait vieilli. Parce que, comme j'ai grandi avec lui, j'ai toujours eu conscience de sa maladie. On dirait qu'une personne avec une DI, quand elle a 4 ou 5 ans, que ça m'affecte moins qu'à l'adolescence. Puis, quand je l'ai regardé, il avait 18 ou 19 ans, et j'ai réalisé l'ampleur de sa déficience. Donc, ça m'a marqué beaucoup ».

\section{Tableau 5}

\section{Perceptions des participants quant à l'influence sur l'éducation à leurs enfants}

\footnotetext{
Extraits de verbatim

"Je veux qu'ils soient en contact avec des gens différents. Justement, qu'ils aient une ouverture d'esprit, qu'ils apprennent à vivre, à partager leur vie avec des gens qui ont des différences. »

"Je pense que les valeurs que je vais transmettre à mes enfants vont être l'acceptation, ne pas juger les gens, des choses comme ça. Je pense que oui, ça va avoir une influence. »

"Il y a beaucoup de monde qui a peur [de la déficience]. Ils ne savent pas comment agir, ils ne veulent pas s'en approcher dans la rue et moi, je ne veux pas que mes enfants aient cette peur-là. »

«Apprendre à vivre un jour à la fois, chaque chose en son temps. D'accepter les différences. »

"Que ce n'est pas nécessairement parce que tu as une DI que tu es limité. Tu as toute une vie devant toi aussi. Donc, c'est de leur transmettre ça : l'intérêt de la différence. »

"C'est sûr que c'est hors de question que j'accepte que mes enfants soient parmi les enfants qui font ce que les autres faisaient à mon frère. Ça, c'est hors de question. "

"J'essaierais de leur faire côtoyer des gens avec une déficience pour qu'ils n'aient pas de préjugés. De s'imprégner jeune de cette façon de vivre qui est différente, mais qui est pas effrayante. »
}

Du côté des évènements positifs, 4 participants mentionnent des évènements s'étant produits à l'école : «À l'école, j'avais un statut privilégié parce que j'avais un frère différent». Trois soulignent l'implication de la personne présentant une DI dans des activités et 3 autres mentionnent des interactions positives dans la fratrie et la famille. Trois indiquent également l'aspect positif des apprentissages de la personne présentant une DI :
«J'avais 12-13 ans, il devait avoir 5-6 ans, et c'était la première fois que j'entendais mon frère dire mon nom au complet, comme il faut. Les autres autour de moi, ils ne comprenaient pas pourquoi j'avais la larme à l'œil, mais... pour moi, c'était important $q u$ 'il sache dire mon nom ». Enfin, 2 participants se rappellent des évènements positifs au plan social. Un de ceux-ci explique : "Moi, parler à du monde que je ne connais pas... à l'école on nous a appris de pas

Volume 23, 2012 
parler aux inconnus. Mon frère il ne s'est pas limité à ça. Il va chercher la collaboration des gens. Il n'a pas peur de demander. Ça, c'est une autre leçon de vie qui peut nous emmener loin dans la vie».

\section{$\underline{\text { Les besoins de soutien }}$}

La septième partie de l'entrevue cible le besoin de soutien perçu et souhaité et l'utilisation de services. Seuls 2 participants croient avoir besoin d'aide dans leur rôle. Des 11 ne croyant pas avoir besoin d'aide, 3 mentionnent la possibilité d'en avoir besoin plus tard, lorsque leurs parents ne seront plus en mesure de répondre aux besoins de la personne présentant une DI : «J'imagine que si la journée arrivait où mes parents n'étaient plus là, je pense que j'irais chercher de l'aide sur comment m'en occuper sur une base constante ». L'aide dont les participants croient avoir besoin, actuellement ou dans le futur, se situe au plan des services formels à la suite du décès de leurs parents $(n=3)$, d'un soutien amical $(n=2)$ et de discussions avec d'autres personnes dans la même situation qu'eux $(n=2)$.

\section{$\underline{\text { Tableau } 6}$}

Influences d'être le frère ou la sœur d'une personne présentant une déficience intellectuelle

\begin{tabular}{|c|c|c|c|c|c|c|c|c|}
\hline \multirow[b]{2}{*}{ 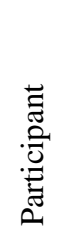 } & \multirow[b]{2}{*}{ 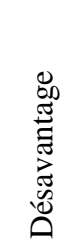 } & \multirow[b]{2}{*}{ 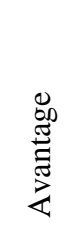 } & \multirow[b]{2}{*}{ 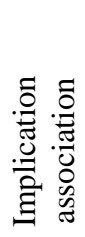 } & \multicolumn{5}{|c|}{ Perception d'une influence sur les projets de vie } \\
\hline & & & & $\begin{array}{l}\text { Choix de } \\
\text { carrière }\end{array}$ & $\begin{array}{l}\text { Lieu de } \\
\text { résidence } \\
\text { actuel }\end{array}$ & $\begin{array}{l}\text { Lieu de } \\
\text { résidence } \\
\text { futur }\end{array}$ & $\begin{array}{c}\text { Désir } \\
\text { d'avoir des } \\
\text { enfants }\end{array}$ & $\begin{array}{c}\text { Éducation } \\
\text { aux } \\
\text { enfants }\end{array}$ \\
\hline 1 & Oui & Oui & Oui & Oui & Oui & Non & Non & Oui \\
\hline 2 & Oui & Oui & Oui & Oui & Non & Non & Non & Non \\
\hline 3 & Oui & Oui & Oui & Oui et non & Oui & Oui & Non & Oui \\
\hline 4 & Non & Oui & Oui & Non & Non & Non & Non & Oui \\
\hline 5 & Non & Oui & Non & Oui & Non & Oui & Non & Oui \\
\hline 6 & Non & Oui & Oui & Oui & Non & Oui & Non & Oui \\
\hline 7 & Oui & Oui & Non & Non & Non & Oui & Non & Oui \\
\hline 8 & Oui & Oui & Non & Non & Oui & Oui & Non & Oui \\
\hline 9 & Oui & Oui & Non & Oui & Oui & Oui & Oui & Oui \\
\hline 10 & Non & Oui & Non & Oui et non & Non & Oui & Non & Oui \\
\hline 11 & Oui & Oui & Non & Oui et non & Non & Oui & Non & Non \\
\hline 12 & Oui & Oui & Oui & Oui & Non & Oui & Oui & Oui \\
\hline 13 & Non & Non & Non & Non & Non & Oui & Non & Oui \\
\hline $\begin{array}{c}\text { Total } \\
\text { oui }\end{array}$ & 8 & 12 & 6 & 9 & 4 & 10 & 2 & 11 \\
\hline
\end{tabular}

Deux participants ont déjà utilisé des services pour les soutenir dans leur rôle, tous les deux pendant l'enfance. Les personnes n'ayant jamais utilisé de services expliquent leur choix ainsi : elles vivent dans une famille ouverte à la discussion et leurs parents les informent $(n=6)$, elles n'ont jamais ressenti le besoin d'avoir une aide extérieure à la famille $(n=6)$ ou elles ne connaissent pas les services $(n=2)$.

\section{$\underline{\text { Les suggestions }}$}

La dernière partie de l'entrevue concerne les suggestions aux frères et aux sœurs, aux parents et aux dispensateurs de services. La majorité des suggestions aux frères et aux sœurs concerne les ressources $(n=8)$. Les participants mentionnent, entre autres, la nécessité de s'informer et de demander de l'aide. Cinq exposent l'importance de l'acceptation de la DI par la fratrie et 3 soulignent la 
nécessité de créer des réseaux sociaux de personnes partageant un même vécu, soit par des forums sur Internet ou en organisant des rencontres avec d'autres familles.

Pour les parents, 8 participants recommandent d'être équitables entre les enfants. Cela implique de faire le moins de différence possible entre les enfants et de garder des moments privilégiés avec la personne ayant un développement typique. Quatre suggèrent que les parents ont le devoir d'aller chercher les ressources et les informations pour leurs enfants. Enfin, 2 soulignent l'importance de parler ouvertement de la DI : «Avoir une discussion plus ouverte sur qu'est-ce que ça implique puis sur le futur aussi. Peut-être pas au début, mais une fois que les enfants ont atteint un certain âge ».

Les suggestions concernant les services ciblent surtout l'apport d'une aide informationnelle $(n=4)$ ainsi que l'augmentation des services et leur facilité d'accès $(n=4)$. Les 2 participants habitant une région éloignée font partie des 4 qui souhaitent que leur famille ait accès à davantage de services. Deux suggèrent également qu'il y ait plus de publicité pour ces services. Enfin, 2 participants encouragent l'organisation de rencontres avec d'autres familles d'enfant ayant une DI.

\section{DISCUSSION}

Cette étude avait pour objectif d'explorer les perceptions des frères et des sœurs adultes d'une personne présentant une DI : influence de cette personne sur leurs projets de vie, perceptions des caractéristiques de la personne ayant une DI, perceptions des relations fraternelles dans la famille et, le cas échéant, perceptions du soutien souhaité. Cette étude étant descriptive et de nombreux éléments ayant déjà été présentés dans les résultats, la conclusion fait le lien entre les éléments saillants des entrevues et la littérature existante à ce sujet. Elle explore également les suggestions des participants ainsi que les besoins exprimés.

\section{L'implication des frères et des sœurs : une aide importante}

Les participants semblent très impliqués auprès de leur famille. En effet, 10 sur 13 sont en contact direct, une fois par semaine ou plus, avec la personne présentant une DI. Ces résultats correspondent à ceux de l'étude de Seltzer et al. (1991) qui indique que $75,6 \%$ des fratries ont des contacts hebdomadaires. Cependant, l'étude de Seltzer est réalisée auprès des frères et des sœurs les plus impliqués auprès de la personne présentant une DI. Il est donc envisageable que les participants de la présente étude soient également ceux les plus impliqués dans la fratrie, en partie puisque l'implication dans l'étude est volontaire. Par exemple, 6 participants ont un rôle formel de tuteur ou de fiduciaire et des 7 autres, 5 croient avoir des responsabilités différentes étant donné que leur frère ou leur sœur présente une DI.

L'implication des participants envers leur fratrie est incontestable. Toutefois, l'aide offerte est davantage d'ordre fonctionnel (aide matérielle et concrète) qu'affectif (aide émotionnelle), bien que 7 d'entre eux soulignent l'amour fraternel comme raison de se rencontrer. Taylor et al. (2008) font un constat similaire. Selon ces auteurs, les frères et les sœurs résident près et vont souvent rendre visite aux personnes présentant une DI, mais ils discutent moins avec eux que ne le font entre eux les frères et les sœurs sans déficience. Ces chercheurs affirment donc que les frères et les sœurs apportent beaucoup d'aide, mais sont affectivement moins proches de leur famille d'origine. Ainsi, les relations se caractérisent davantage par l'obligation que par un sentiment de proximité. Étant donné leur rôle de premier aidant auprès des parents, et parfois de relève à ceux-ci, il apparaît important de soutenir les frères et les sœurs dans leur rôle, particulièrement au moment où leurs responsabilités augmentent. Ils doivent, entre autres, avoir de l'information quant au réseau de services.

Les besoins des frères et des sœurs : la place des parents et des services

La façon dont les parents vivent le diagnostic a aussi une influence. Entre autres, 7 participants disent voir la DI de la même manière que les parents et plusieurs expliquent qu'étant donné que leurs parents acceptent bien la DI, ils ont appris à l'accepter aussi. Il apparaît donc important d'informer les parents de leur influence à ce sujet. Les parents devraient avoir un soutien pour la compréhension et l'acceptation du diagnostic. Ce

Volume 23, 2012 
soutien leur permettrait d'assister leurs autres enfants sur ce plan.

La majorité des participants soulignent que les attentes de leurs parents à leur égard correspondent aux leurs. Pour plusieurs, ces attentes ont fait l'objet de discussions entre eux. Par contre, pour 3 participants, les attentes de leurs parents sont encore inconnues, particulièrement en ce qui concerne l'avenir. Les études au sujet de la planification pour le futur semblent démontrer que le transfert de la responsabilité de la personne présentant une DI, à la suite du décès ou de l'incapacité des parents à répondre à ses besoins, ne fait pas fréquemment l'objet d'une planification (Bernard et Goupil, 2012). Ainsi, c'est souvent dans un contexte de crise qu'on redistribue les responsabilités (Freedman, Krauss et Seltzer, 1997; Pruchno, Patrick et Burant, 1996). Une discussion plus ouverte sur le sujet aiderait les membres de la famille à s'engager dans un processus commun concernant la planification de leur futur.

Les participants n'utilisent pas les services formels pour les soutenir ou pour obtenir l'information dont ils ont besoin. Cela s'explique, du moins en partie, parce que ce sont les parents qui transmettent les renseignements relatifs à la personne présentant une DI aux autres membres de la famille. Les parents semblent le faire d'une manière informelle et dévoilent l'information jugée essentielle. À ce sujet, les parents pourraient profiter d'une aide informationnelle pour les assister dans leurs explications. Un autre point soulevé par les participants est leur méconnaissance des services. Selon le ministère de la Santé et des Services sociaux (2001), les différents membres de la famille, dont la fratrie, sont la clientèle visée pour un soutien. À ce sujet, le Processus clinique montréalais, élaboré par les CRDITED de Montréal (2009) indique que les membres de la famille peuvent bénéficier d'une assistance éducative en vue de bien comprendre la personne présentant une DI. Certains auteurs ont déjà démontré que les frères et les sœurs d'une personne présentant une DI ont besoin d'information (Heller et Kramer, 2009; Krauss et al., 1996) et qu'il est nécessaire de développer des services répondant à leurs besoins (Seltzer et al., 1997; Williams, Piamjariyakul, Graff et Stanton, 2010). À ce propos, 3 participants proposent la création de réseaux sociaux, virtuels ou en personne, pour les frères et les sœurs. Deux autres participants indiquent que des échanges avec des familles vivant la même situation, entre autres par l'entremise des associations, sont un bon moyen de mieux comprendre la DI et de développer un réseau d'entraide et d'écoute.

Les influences : désagréments, avantages et projets de vie

Selon nos résultats, les participants vivent certains désagréments. Entre autres, ils regrettent de ne pas expérimenter de relations fraternelles normales et trouvent difficile d'être identifiés comme « le frère ou la sœur d'une personne présentant une DI». Grossman (1972), dans une des premières études s'intéressant à ces fratries $(n=83)$, concluait déjà à des effets négatifs chez $46 \%$ d'entre eux, en comparaison aux frères et sœurs d'une personne ayant un développement typique $(n=66)$. Ces personnes ressentaient de la honte, de la colère, de la culpabilité, des perturbations familiales et un manque d'attention parentale. Les résultats de Grossman ont été validés par des études subséquentes (Burton et Parks, 1994; Fahey, 2005). Ces résultats soulignent l'importance de considérer, même à l'âge adulte, les conséquences psychologiques vécues par les fratries. À cet effet, 8 participants mentionnent l'importance d'être équitable envers les enfants, même devenus adultes. Selon eux, les parents doivent garder des moments privilégiés avec leurs autres enfants. Aussi, ils ne devraient pas leur imposer de trop grandes responsabilités en lien avec les soins à la personne présentant une DI.

Du côté des avantages, 8 participants soulignent l'influence de la personne ayant une DI sur leur ouverture d'esprit ainsi que leurs occasions de croissance personnelle. Cet aspect a aussi été mis en évidence par Grossman (1972) et Burton et Parks (1994). Ces auteurs s'entendent, entre autres, sur les aspects de tolérance, de détermination et de compréhension des différences développées par les frères et les sœurs d'une personne présentant une DI. De plus, les participants de la présente étude disent profiter pleinement des joies de la vie quotidienne et faire des rencontres grâce à la personne ayant une DI. En général, cette expérience apparaît comporter à la fois des éléments positifs et négatifs. Il est intéressant qu'aucun des participants ne mentionne 
uniquement des composantes négatives, cela est peut-être dû à leur grande implication ou à la désirabilité sociale.

Concernant l'influence sur leurs projets de vie, 9 participants sur 13 perçoivent que la présence de la personne présentant une DI influence leur orientation professionnelle. La littérature disponible à ce sujet est partagée. Plusieurs auteurs concluent à des différences non significatives quant à l'orientation professionnelle entre des groupes de frères et de sœurs d'une personne ayant une DI et des groupes de frères et de sœurs d'une personne ne présentant pas de déficience (Burton et Parks, 1994), un TED (Orsmond et Seltzer, 2007) ou ayant un trouble de santé mentale (Seltzer et al., 1997). D'autres chercheurs soulignent qu'il y a une influence sur la fratrie (Mishel, 2004; Seltzer et al, 1997). À ce sujet, Mishel rapporte, dans une étude effectuée auprès de 16 professionnels de la santé ayant un frère ou une sœur ayant une déficience, que 6 occupent un emploi en lien avec le type de déficience de leur frère ou leur sœur. En se fondant sur son expérience clinique, Scelles (1997) soutient que les fratries choisissent plus souvent des carrières médico-sociales.

En ce qui concerne le choix du lieu de résidence, les réponses des participants semblent refléter leur âge (20 à 35 ans). En effet, 4 sont aux études et plusieurs considèrent encore la possibilité de déménager. À ce stade-ci de leur vie, ces réponses indiquent que la présence de la personne présentant une DI ne les influence pas sur ce plan. Par contre, plusieurs frères ou sœurs de la présente étude croient que la DI aura une influence sur leurs futurs déplacements, après le décès de leurs parents. Enfin, peu de participants $(n=2)$ perçoivent une influence sur leur désir d'avoir des enfants, mais plusieurs $(n=11)$ entrevoient une influence sur leur façon de les éduquer.

\section{Les limites de l'étude et les recherches futures}

Les résultats de cette étude exploratoire, obtenus à partir d'un seul questionnaire, doivent faire l'objet d'interprétations en tenant compte de ses limites. Tout d'abord, la taille et la provenance du groupe, soit 13 participants québécois, ne permettent pas de généraliser les résultats à l'ensemble des frères et des sœurs d'une personne présentant une DI. De plus, 9 sur 13 ont fait des études universitaires, ce qui ne constitue pas le niveau de scolarité moyen de la population (Statistique Canada, 2008). Il serait approprié de mener des études incluant plus de participants et représentant mieux la population générale. Ces études pourraient également comparer les frères et les sœurs en fonction de leur implication présente et future et de leurs sentiments face à leur famille, ce que la présente étude ne s'est pas attardée à faire.

Les personnes présentant une DI dont il est question en entrevue présentent des caractéristiques différentes. Certaines ont des déficiences physiques associées, des troubles de santé ou un syndrome particulier, tel le syndrome de Down. Cette hétérogénéité constitue une autre limite étant donné que les caractéristiques associées à ces conditions peuvent avoir une influence sur les relations fraternelles. Des études interrogeant des fratries de personnes présentant des caractéristiques plus homogènes et tentant de différencier l'influence de ces conditions sont envisageables.

Les participants sont tous des volontaires ayant répondu à une annonce. Il est possible que le mode de recrutement influence le type de personnes collaborant à l'étude. Les personnes vivant moins bien les relations fraternelles peuvent ne pas avoir été intéressées à collaborer. En effet, les participants s'impliquent beaucoup auprès de la personne ayant une DI et plusieurs œuvrent dans des associations. Des études auprès de fratries moins impliquées sont souhaitables afin de comprendre comment ces dernières perçoivent leur situation familiale. Ensuite, puisque la méthode employée inclut des entrevues, il est possible qu'il y ait un biais lié à la désirabilité sociale.

Finalement, cette étude explore les besoins des personnes uniquement âgées de 20 à 34 ans. Plusieurs participants ont souligné que leurs besoins vont varier avec le temps et s'attendent à une augmentation de leurs responsabilités dans l'avenir. Davys, Mitchell et Haigh (2011) indiquent d'ailleurs que les rôles de la fratrie fluctuent avec le cycle de vie de la famille. Des études sur les autres étapes de vie, sur leur engagement futur et sur la planification du transfert des responsabilités des personnes ayant une DI sont souhaitables. 


\section{CONCLUSION}

Les résultats de la présente étude permettent d'avoir une meilleure compréhension de la dynamique existant dans les fratries et les familles dans lesquelles il y a une personne présentant une DI. Cela suscite une plus grande ouverture d'esprit, mais apporte aussi des défis particuliers. Les fratries s'impliquent assidument et les participants croient que leur implication diffère de celle des fratries typiques. De plus il semble qu'être le frère ou la sœur d'une personne ayant une DI influence plusieurs aspects des projets de vie, par exemple, les choix de carrière pour certains participants ou les déplacements futurs loin du domicile des parents. Les résultats mettent en évidence l'importance de l'information sur les services. Il semble ainsi essentiel d'outiller les familles afin qu'elles ne se sentent pas dépourvues et qu'elles aillent chercher le soutien nécessaire au bien-être de leurs enfants.

\title{
PERCEPTIONS OF ADULT SIBLINGS OF A PERSON WITH INTELLECTUAL DISABILITY ON THEIR MUTUAL RELATIONS IN THE FAMILY, THEIR LIFE PROJECTS AND THE EXPECTED SUPPORT
}

\begin{abstract}
Few studies have examined the relationships among siblings when an adult member has an intellectual disability (ID). This study aims to explore the perceptions of the adult brothers and sisters of a person with an intellectual disability on the influence of the disability on the sibling's life projects. It also aims to describe the sibling's perceptions on their brother or sister behaviors and skills, relationship in the family and the expected support in this context. Thirteen adult siblings of a person with an ID participated in an interview. Analysis revealed that participants are involved in family life by supporting their parents and their brother or sister with an ID. Many participants mentioned that their brother or sister's disability affects their life plans, particularly their plans for career, their future place of residence and their children's education. Finally, clinical recommendations regarding support for the families are presented.
\end{abstract}

\section{BIBLIOGRAPHIE}

Ansen-Zeder, E. (2010). Alterite traumatique, adaptation, résilience être frère ou soeur d'une personne en situation de handicap mental (Thèse de doctorat). Récupéré de http://artur.univ-

fcomte.fr/SLHS/PSYCHO/these/these_zedere lisabeth.pdf

Bernard, M.-A. (2011). Transition en cours de vie de personnes ayant une déficience intellectuelle : recherche exploratoire sur l'après-parents (essai doctoral non publié). Université du Québec à Montréal, Montréal, Canada.

Bernard, M.-A., Goupil, G. (2012). L'après-parents : étude exploratoire sur les perceptions de mères qui vieillissent avec un adulte ayant une déficience intellectuelle. La Revue canadienne du vieillissement, 31, 85-72.
Burke, M. M., Taylor, J. L., Urbano, R., Hodapp, R. M. (2012). Predictors of future caregiving by adults siblings of individuals with intellectual and developmental disabilities. American Journal of Intellectual and Developmental Disabilities, 117, 33-47.

Burton, S. L., Parks, A. L. (1994). Self-esteem, locus of control, and career aspirations of collegeage siblings of individuals with disabilities. Social Work Research, 18, 178-185.

Cicirelli, V. G. (1995). Strengthening sibling relationships in the later years. In G. C. Smith, S. S. Tobin, E. A. Robertson-Tchabo, P. W. Power (Eds.). Strengthening Aging Families : Diversity in Practice and Policy (pp 45-60). Thousand Oaks, CA : Sage Publications. 
Coniglio, F., Meldrum, D. (2011). MIFA siblings project. Workshop presented at the Mental Illness Fellowship of Australia Inc. Récupéré de

http://www.mifa.org.au/sites/www.mifa.org.a $\mathrm{u} /$ files/documents/MIFA\%20Sibling\%20The MHS\%20Conference.pdf

Davys, D., Mitchell, D., Haigh, C. (2011). Adult sibling experience, roles, relationship and future concerns - a review of the literature in learning disabilities. Journal of Clinical Nursing, 20, 2837-2853.

Doody, M. A., Hastings, R. P., O'Neill, S., Grey, I. M. (2010). Sibling relationships in adults who have siblings with or without intellectual disabilities. Research in Developmental Disabilities, 31, 224-231. doi : 10.1016/j.ridd.2009.09.007

Fahey, A. (2005). Psychological adjustment of siblings of adults with and without mental retardation. Dissertation Abstracts International, 66 (5-B). (UMI no. 3177109)

Freedman, R. I., Krauss, M. W., Seltzer, M. M. (1997). Aging parents' residential plans for adult children with mental retardation. Mental Retardation, 35, 114-123. Récupéré de http://www.waisman.wisc.edu/family/pubs/A gingDD/8\%20\%20MR_1997_aging_parents_ residential.pdf

Fujiura, G. T. (2010). Aging families and the demographic of family financial support of adults with disabilities. Journal of Disability Policy Studies, 20, 241-250. doi : $10.1177 / 1044207309350560$

Griffiths, D. L., Unger, D. G. (1994). Views about planning for the future among parents and siblings of adults with mental retardation. Family Relations, 43, 221-227.

Grossman, F. K. (1972). Brothers and sisters of retarded children : An exploratory study. Oxford, England : Syracuse University Press.

Heller, T., Kramer, J. (2009). Involvement of adult siblings of persons with developmental disabilities in future planning. Journal of Intellectual \& Developmental Disability, 47, 208-219. doi : 10.1352/1934-9556-47.3.208

Hodapp, R. M., Glidden, L. M., Kaiser, A. P. (2005). Siblings of persons with disabilities : Toward a research agenda. Mental Retardation, 5, 334-338. Récupéré de http://www.aaiddjournals.org/doi/pdf/10.1352 /00476765\%282005\%2943\%5B334\%3ASSO SEB\%5D2.0.CO\%3B2

Holmes, N., Carr, J. (1991). The pattern in care of families of adults with a mental handicap: A comparison between families of autistic adults and Down Syndrome adults. Journal of Autism and Developmental Disorders, 21, 159-176.

Kao, B., Romero-Bosch, L., Plante, W., Lobato, D. (2011). The experiences of Latino siblings of children with developmental disabilities. Child : Care, Health and Development, 38, 545-552.

Krauss, M. W., Seltzer, M. M., Gordon, R., Friedman, D. H. (1996). Binding ties : The roles of adult siblings of persons with mental retardation. Mental Retardation, 34, 83-93.

Les centres de réadaptation en déficience intellectuelle et troubles envahissants du développement de Montréal (2009). Processus clinique montréalais. Montréal, Canada. Récupéré de : http://www.crgm.qc.ca/cm/images/stories/doc uments/processus \%20clinique\%20montralais. pdf

Luckasson, R., Borthwick-Duffy, S., Bintinx, W. H. E., Coulter, D. L., Craig, E. M., Reeve, A... Tasse, M. J. (2002/2003). Retard mental: définition, classification et systèmes de soutien. Washington, DC : American Association on Mental Retardation.

Miles, M. B., Huberman, A. M. (2003). Analyse des données qualitatives, traduction de la $2^{\mathrm{e}}$ édition américaine. Bruxelles, Belgique : De Boeck.

Volume 23, 2012 
Ministère de la Santé et des Services sociaux. (2001). De l'intégration sociale à la participation sociale : Politique de soutien aux personnes présentant une déficience intellectuelle, à leurs familles et aux autres proches. Récupéré de

http://publications.msss.gouv.qc.ca/acrobat/f/ documentation/2001/01-840-01.pdf

Mishel, L. A. (2004). Exploring the career motivation of health professionals : Impact of experience with an ill or handicapped sibling. Dissertation Abstracts International. (UMI no. 3139796)

Oris, M., Brunet, G., Widmer, E., Bideau, A. (2007). Les fratries: une démographie sociale de la germanité. Berne, Allemagne : Peter Lang.

Orsmond, G. I., Seltzer, M. M. (2000). Brothers and sisters of adults with mental retardation : Gendered nature of the sibling relationship. American Journal on Mental Retardation, 105, 486-508. Récupéré de http://www.waisman.wisc.edu/family/pubs/A gingDD/23\%20\%20AJMR_2000_brothers_si sters.pdf

Orsmond, G. I., Seltzer, M. M. (2007). Siblings of individuals with autism or Down syndrome: Effects on adult lives. Journal of Intellectual Disability Research, 51, 682-696. doi : 10.1111/j.1365-2788.2007.00954.x

Pruchno, R. A., Patrick, J. H., Burant, C. J. (1996). Aging women and their children with chronic disabilities : Perceptions of sibling involvement and effects on well-being. Family Relations, 45, 318-326. Récupéré de http://links.jstor.org/sici?sici=01976664\%28199607\%2945\%3A3\%3C318\%3AA WATCW\%3E2.0.CO\%3B2-A\&

Rizzolo, M. C., Hemp, R., Braddock, D., PomeranzEssley, A. (2004). The state of the states in developmental disabilities : 2004. Boulder, CO : University of Colorado, Coleman Institute for Cognitive Disabilities and Department.
Scelles, R. (1997). Le sens du choix d'une profession médico-sociale pour les frères et sœurs de personnes porteuses de handicap. Revue francophone de la déficience intellectuelle, 8 , 103-113. Récupéré de http://www.rfdi.org/files/SCELLES-vol8no2103-113.PDF

Scelles, R. (2007). Le sens du choix d'une profession médico-légale pour les frères et les sœurs de personnes en situation de handicap. Revue francophone de la déficience intellectuelle, 8 , 103-113.

Seltzer, G. B., Begun, A., Seltzer, M. M., Krauss, M. W. (1991). Adults with mental retardation and their aging mothers: Impacts of siblings. Family Relations, 40, 310-317. Récupéré de http://www.jstor.org/stable/585017

Seltzer, M. M., Greenberg, J. S., Krauss, M. W., Gordon, R. M., Judge, K. (1997). Siblings of adults with mental retardation or mental illness: Effects on lifestyle and psychological well-being. Family Relations. Special Issue: Family Care Giving For Persons With Disabilities, 46, 395-405. Récupéré de http://psychservices.psychiatryonline.org/cgi/r eprint/50/9/1214

Seltzer, M. M., Greenberg, J. S., Orsmond, G. I., Lounds, J. (2005). Life course studies of siblings of individuals with developmental disabilities. Mental Retardation, 43, 354-359. Récupéré de http://www.waisman.wisc.edu/family/pubs/A utism/2005\%20Life $\% 20$ course $\% 20$ study $\% 20$ sibs $\% 20$ of $\% 20$ indv $\% 20$ with $\% 20$ dd.pdf

Statistique Canada, Division de la statistique sociale et autochtone. (2008). Enquête sur la participation et les limitations d'activités de 2006 : L'expérience de travail des personnes avec incapacités au Canada. Ottawa, Canada : Gouvernement du Canada. Récupéré de http://www.statcan.gc.ca/pub/89-628-x/89628-x2008007-fra.pdf

Taylor, J. L., Greenberg, J. S., Seltzer, M. M., Floyd, F. J. (2008). Siblings of adults with mild intellectual deficits or mental illness: 
differential life course outcomes. Journal of Family Psychology, 22, 905-914. doi : $10.1037 / \mathrm{a} 0012603$

Turnbull, A., Turnbull, R., Erwin, E. J., Soodak, L. C. (2006). Families. professionals, and exceptionality. Positive outcomes through partnerships and trust - 5th ed. Columbus, $\mathrm{OH}$ : Prentice Hall.

Villeneuve, M., Goupil, G., Gascon, H. (2011). Relations à l'âge adulte entre les personnes présentant une déficience intellectuelle et leur fratrie. Revue francophone de la déficience intellectuelle, 22, 98-111.
Williams, D. P., Piamjariyakul, U., Graff, J. C., Stanton, A. (2010). Developmental disabilities : Effects on well-sibling. Issues in Comprehensive Pediatrics Nursing, 33, 3955. doi : 10.3109/01460860903486515

Wilson, K. R. (2011). An exploration of the relationships between young adult women and their sisters with intellectual disabilities (Master's thesis). Retiré de https://dspace.smith.edu/bitstream/handle/110 20/23018/Katharine\%20R.\%20Wilson$\% 20$ Final $\% 20$ Thesis.pdf?sequence $=1$

Zetlin, A. G. (1986). Mentally retarded adults and their siblings. American Journal of Mental Deficiency, 91, 217-225. 